\title{
Konzeption und Entwicklung eines Smart- Glasses-basierten Informationssystems zur Montage und Bestückung von Verkaufsdisplays
}

Laura Sophie Gravemeier, Steffen Beermann, Dennis Meyer zu Bergsten, Christian Kaiser und Oliver Thomas

Im Rahmen des GLASSHOUSE-Projekts wurde prototypisch ein Informationssystem zur Unterstützung eines Value-Added-Service-Prozesses für ein Logistikunternehmen entwickelt. Dabei übernimmt das Logistikunternehmen die Montage und Bestückung von Verkaufsdisplays, die als verkaufsfördernde Maßnahme im Einzelhandel zur ansprechenden Präsentation der Ware eingesetzt wird. Basierend auf bereits etablierten Design-Richtlinien wurde das System für die Smart Glasses M300 von Vuzix konzipiert und in einem iterativen Entwicklungsprozess umgesetzt. Dabei wurde das User Interface an die Anforderungen und Limitationen von Smart Glasses angepasst sowie eine intuitive und freihändige Bedienung via Sprachsteuerung umgesetzt. Auf die Konzeption und Implementierung wird in den folgenden Abschnitten detailliert eingegangen.

\section{Einleitung}

In diesem Beitrag wird die Konzeption und Entwicklung eines Informationssystems beschrieben, das beispielhaft die Führung durch informationsintensive Prozesse mit Smart Glasses im Rahmen des GLASSHOUSE-Projekts umsetzt. Konkret wird eine Anwendung für einen Value Added Service in der Logistik, die Montage und die Befüllung von Verkaufsdisplays, mit einem Smart-Glasses-basierten Informationssystem vorgestellt. Ein Verkaufsdisplay dient dabei der Präsentation der Ware im Einzelhandel zur Verkaufsförderung und wird insb. bei Saisonartikeln angewendet (Horstmann und Lingenfelder 2015).

Zunächst werden die technischen Rahmenbedingungen beim Einsatz von Smart Glasses beschrieben. Daraufhin werden die etablierten Designgrundlagen für das User Interface (UI) von Smart Glasses erläutert. Anschließend werden das Konzept des Informationssystems erarbeitet, die prototypische Umsetzung detailliert vorgestellt und vorhandene Limitationen diskutiert. Abschließend werden die Entwicklung des Informationssystems reflektiert und ein Ausblick gegeben. 


\section{Technische Rahmenbedingungen}

Smart Glasses sind Wearables, die als Head-Mounted-Display getragen werden und zusätzliche Informationen direkt im Sichtfeld des Nutzers anzeigen (Zobel et al. 2018). Sie verfügen über verschiedene Sensoren wie Kamera, Mikrofon, Lagesensoren und die Möglichkeit der kabelgebundenen und drahtlosen Kommunikation.

Das Einsatzgebiet von Smart Glasses fokussiert sich aktuell auf den Industriesektor. Mit diesem Fokus sind im Laufe der Jahre mehrere Hersteller mit eigenen Produkten am Markt erschienen. Dazu zählt bspw. Vuzix mit der M100 und deren Nachfolgemodell, der M300.

\subsection{Ausstattung der M300}

Im Rahmen des GLASSHOUSE-Projekts wurde das im Jahr 2017 veröffentlichte Modell M300 der Firma Vuzix verwendet. Die in Abb. 1 dargestellte M300 besteht aus einem externen Akku und einer Recheneinheit, an welcher das Display angebracht ist. Diese Bestandteile werden an jeweils einer Seite des mitgelieferten Brillengestells angebracht, wobei ihre Platzierung frei wählbar ist. Das Display hat eine HD-Auflösung und ein Sichtfeld von $20^{\circ}$. Die Anzeigegröße entspricht ungefähr einem fünf Zoll großen Display in einer Entfernung von 45 Zentimetern. In der M300 sind ein Dual Core Intel Atom CPU und 2 GB Arbeitsspeicher verbaut. Es stehen ein Mikrophon mit Geräuschunterdrückung, ein Lautsprecher und eine 10 Megapixel Kamera zur Verfügung, welche mit 1080p Videos aufnehmen kann. Die M300 kann je nach Nutzungsgrad zwischen 2 und 12 Stunden mit einer Akkuladung betrieben werden. Der externe Akku ist im laufenden Betrieb austauschbar. Zur Konnektivität stehen WiFi sowie Bluetooth 4.1 zur Verfügung.

Auf der M300 läuft eine angepasste Version des Betriebssystems Android in Version 6.0.1. Damit ist die Kompatibilität mit existierenden Apps für diese Plattform grundsätzlich gewährleistet. Vuzix bietet für die M300 ein Software-Development-Kit (SDK) an, welches Application Programming Interfaces (API) zur Sprachsteuerung sowie Barcodeerkennung bereitstellt.

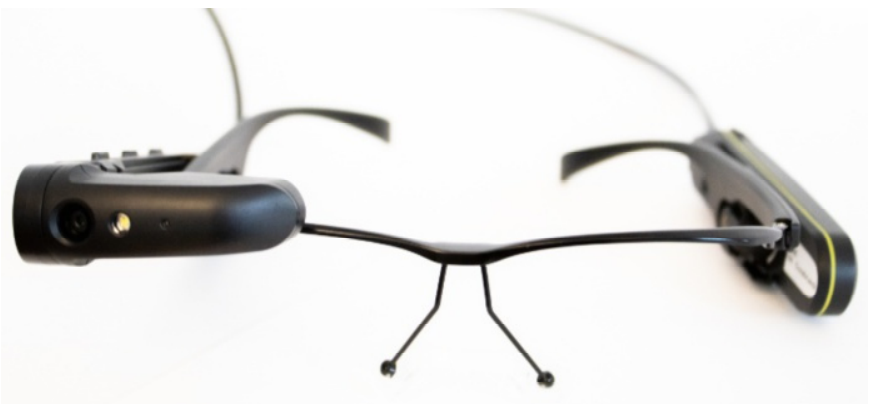

Abb. 1. Vuzix M300 Smart Glasses 


\subsection{Interaktionsmöglichkeiten}

Zur Interaktion bietet die M300 verschiedene Schnittstellen. Bei diesen handelt es sich um Sprachsteuerung sowie drei Hardware-Tasten und ein Touchpad an der Recheneinheit der Datenbrille. Die Hardware-Tasten beinhalten insg. sechs Funktionen, welche sich durch kurzes Drücken oder längeres Halten ausführen lassen. Das Touchpad registriert horizontale und vertikale Bewegungen, wodurch entsprechende Navigation oder das Interagieren mit Schiebereglern möglich ist. Bei der Sprachsteuerung verwendet Vuzix eine Eigenentwicklung, welche durch den Sprachbefehl „Hello Vuzix“ aus dem Ruhemodus geweckt wird und daraufhin einen Befehl entgegennehmen kann. Dabei ist sie darauf ausgelegt, einzelne und kurze Anweisungen zu registrieren und nicht ganze Sätze zu interpretieren.

\section{Designgrundlagen des User Interfaces für Smart Glasses}

Zu Beginn der Entwicklung des Prototyps wurde ersichtlich, dass bislang wenige Implementierungen für Anwendungen auf Smart Glasses existieren. Dies lässt darauf schließen, dass diese Form der Endgeräte noch keine abschließende Marktreife erreicht hat. Entsprechend gibt es nur wenige Informationen, wie das UI für Smart Glasses zu designen ist. Dieser Abschnitt stellt bereits veröffentlichte Vorgaben dar, die im Rahmen des Designs von UI für Smart Glasses zu beachten sind.

Google hat mit der Veröffentlichung der Google Glass eine Guideline zum Design von Anwendungen herausgegeben, welche jedoch auf dieses konkrete Endgerät bezogen und daher nur eingeschränkt übertragbar sind. In dieser wurden die allgemeinen Prinzipien (P1) ,Don't get in the way“, (P2) „Keep it relevant“, (P3) „Avoid the unexpected“ sowie (P4) „Build for people” aufgestellt, die während des gesamten Design-Prozesses berücksichtigt werden sollen (Google Inc. 2015). Durch (P1) und (P2) soll gewährleistet werden, dass nur die benötigten Informationen zum benötigten Zeitpunkt bereitgestellt werden und die Anzeige ansonsten unauffällig bleibt. Da Smart Glasses nah vor dem Auge getragen werden, können unerwartete Anzeigen unangenehm wahrgenommen werden und sollten daher vermieden werden (P3). Durch das vierte Prinzip (P4) soll der Fokus einer einfachen und intuitiven Interaktion zwischen Nutzer und Smart Glasses nicht verloren gehen.

Neben diesen Grundprinzipien gibt es einige konkrete Anforderungen an das UI-Design für Smart Glasses. So hat sich gezeigt, dass die innere, zur Nase gerichtete Bildschirmhälfte sowie der untere Bildschirmbereich besser erkannt und abgelesen werden können (Google Inc. 2015; Tanuma et al. 2011). Es bietet sich daher an, den wichtigen Inhalt in diesem Bereich anzuzeigen. Damit der Bildschirm der Smart Glasses sich besser in das Sichtfeld des Trägers integriert, sollte ein dunkler Hintergrund gewählt werden, auf dem der Inhalt mit einem hohen Kontrast angezeigt wird (Google Inc. 2015; Tanuma et al. 2011; Meta Company 2017). Die 
Schriftgröße sollte dabei für den Inhalt angemessen groß gewählt werden, da dieser sonst schwer lesbar ist (Sony Corporation 2017). Daher sollten die anzuzeigenden Inhalte möglichst minimal gehalten werden, um so lange und komplexe Texte zu vermeiden (Niemöller et al. 2017; Sony Corporation 2017).

\section{Konzeption des Informationssystems}

Im folgenden Abschnitt wird aus den Anforderungen des Anwendungsfalls der Ablauf der Prozessinitialisierung und -führung abgeleitet und dessen Darstellung konzipiert. Abschließend wird das UI unter Berücksichtigung der etablierten Designgrundlagen konzeptionell erarbeitet.

\subsection{Beschreibung des Anwendungsfalls}

Das Informationssystem wurde für den Anwendungsfall einer Prozessführung durch informationsintensive Logistikprozesse entwickelt. Konkret wurde hier ein Value Added Service als Prozess umgesetzt. Dabei handelt es sich um die Mehrwertdienstleistung des Zusammenbaus von Verkaufsdisplays für einen Hersteller, welche später durch den Spediteur an Supermärkte versendet werden. Da die Displays aus vielen Einzelteilen bestehen und verschiedene Exemplare nach unterschiedlichen Vorgaben zu befüllen sind, bedarf es einer detaillierten Anleitung für jeden Einzelfall.

Diese Anleitungen liegen bei dem Logistikunternehmen zu diesem Zeitpunkt in Papierform vor. Der Detaillierungsgrad der Aufgabe führt dazu, dass die Mitarbeiter regelmäßig die Anleitung zur Hand nehmen müssen, um Informationen über die nächsten Prozessschritte zu erhalten. Zudem kann es dazu kommen, dass der Mitarbeiter den Überblick darüber verliert, bei welchem Schritt er sich aktuell befindet und deshalb Zeit dafür aufwenden muss, sich neu zu orientieren. Sowohl Experten als auch Anfänger müssen den Prozess durchlaufen. Dabei steht beiden die gleiche Anleitung zur Verfügung. Der Experte bedarf oft weniger Informationen und lediglich einzelfall-spezifischer Anweisungen. Für Anfänger wiederum beinhaltet die Anleitung zu wenige grundlegende Informationen, da der Zusammenbau nicht detailliert beschrieben wird. So muss bei der Einarbeitung ein erfahrener Mitarbeiter zur Seite stehen.

Die verschiedenen Teilaufgaben des Prozesses werden oft getrennt von unterschiedlichen Mitarbeitern bearbeitet, wobei diese Aufteilung in den Anleitungen nicht berücksichtigt wird. Generell ist bei der Verwendung der Papieranleitungen weder eine Qualitätssicherung noch eine automatische Anbindung an das Enterprise-Resource-Planning-System (ERP-System) gegeben.

Das Informationssystem soll den Anwender durch die einzelnen Schritte des Zusammenbaus sowie die Befüllung des Displays führen. Dabei sollen die oben ausgeführten Defizite der Papieranleitung vermieden und die im Folgenden beschriebenen Anforderungen erfüllt werden. Damit der Mitarbeiter beide Hände 
zum Zusammenbau und Befüllen nutzen kann, wird eine freihändige Bedienung angestrebt (A1: Freihändige Bedienung). Gleichzeitig soll er einen Überblick darüber behalten, bei welchem Schritt er sich derzeit befindet, damit er sich nicht regelmäßig neu orientieren muss (A2: Fortschrittsüberblick). Zusätzlich wird für eine einfachere Navigation die Aufgabe in mehrere Teilaufgaben unterteilt, welche nicht alle von einer Person ausgeführt werden müssen (A3: Aufgaben-/Arbeitsteilung). Die Anweisungen sollen sowohl für Experten als auch für Anfänger angepasst sein, sodass eine differenzierte Aufbereitung der Anweisungen für die Anwendergruppen umgesetzt wird. So werden dem Experten nur die tatsächlich benötigten Informationen angezeigt und die Einarbeitung neuer Mitarbeiter erfordert einen geringeren Aufwand (A4: Kompetenzstufen). Während der Prozessführung werden Abfragen zur Erreichung eines Arbeitsergebnisses gestellt und Materialverfügbarkeiten geprüft. Mit dem Ziel einer integrierten Qualitätssicherung werden abhängig von der Beantwortung entsprechende Folgeschritte angezeigt (A5: Qualitätssicherung).

Im Rahmen dieses Anwendungsfalls soll das Informationssystem den Prozessablauf, im Vergleich zur Papieranleitung, verbessern. Insb. sollen der Zeitaufwand verringert, eine Qualitätssicherung integriert sowie eine Anpassung an die Kompetenz der Mitarbeiter ermöglicht werden. Des Weiteren wäre eine Anbindung an ein ERP-System mithilfe des Informationssystems einfach umsetzbar.

\subsection{Prozessabfolge der Anwendung}

Anhand des beschriebenen Logistikprozesses wurde der in Abb. 2 dargestellte Anwendungsablauf entworfen. Dabei wurde möglichst stark vom konkreten Anwendungsfall abstrahiert. Der Anwendungsablauf wurde in die Abschnitte Prozessinitialisierung und Prozessführung unterteilt.

Innerhalb der Prozessinitialisierung werden Benutzereinstellungen und die Merkmale der Prozessführung festgelegt: Der Benutzer meldet sich an, wählt eine Aufgabe und eine zugehörige Teilaufgabe sowie eine Kompetenzstufe aus. Die darauffolgende Prozessführung umfasst die Leitung durch die einzelnen Arbeitsschritte. Dabei ist eine dynamische Abfolge der Schritte vorgesehen, welche kontextabhängig und durch Benutzereingaben gesteuert wird. Nach Abschluss kann der Benutzer eine weitere Aufgabe auswählen.

Durch die Einschränkungen bei der Verwendung von Smart Glasses ergeben sich besondere Anforderungen an die Darstellung der Arbeitsschritte. Daher sind knappe und elementare Anweisungen nötig, wobei diese verständlich bleiben müssen. Infolgedessen ergibt sich die Notwendigkeit einer geeigneten Darstellung der Arbeitsanweisung gemäß ihrem Zweck. Dabei werden Anweisungen, die ähnliche Elemente enthalten, zu einem Darstellungstyp zusammengefasst. Auf Grundlage der Papieranleitungen und iterativen Evaluationsergebnissen wurden verschiedene Anforderungen an die Prozessführung und angemessene Darstellungstypen abgeleitet: 
- Einfache Textanweisungen, die in der Regel nur für die Ankündigung eines neuen Arbeitsabschnitts verwendet werden.

- Bilder mit Textanweisung, die den grundlegenden Aufbau regulärer Prozessschritte darstellen.

- Bilder mit Textanweisung und Kontrollfunktion, worin zusätzlich die Bestätigung des ausgeführten Schrittes ermöglicht wird.

- Textanweisung mit Scan- oder anderer Dokumentationsfunktion, womit verschiedene Arten der Qualitätskontrolle umgesetzt werden können.

Die konkrete Umsetzung des Anwendungsablaufs und der Darstellungstypen der Prozessführung wird in Abschnitt 5 näher erläutert.

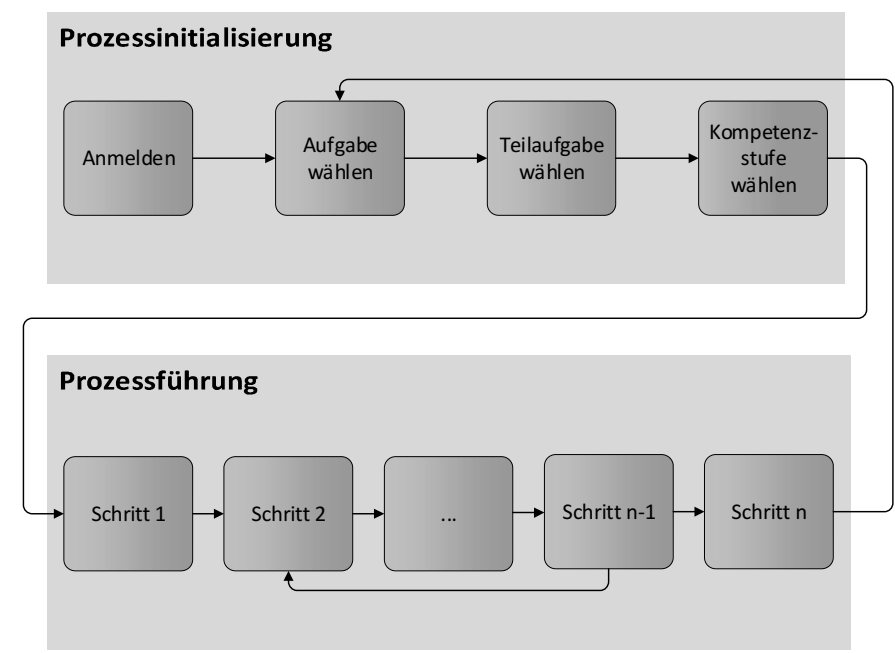

Abb. 2. Anwendungsablauf

\subsection{Design des User Interface}

Das Design der Benutzeroberfläche soll die in Abschnitt 3 beschriebenen Guidelines und Handlungsempfehlungen umsetzen. Ausgerichtet an den Bedürfnissen der Anwender (P4) sollen die Interaktion und Informationsvermittlung möglichst kompakt (P2) und einheitlich (P3) umgesetzt werden, wobei die effiziente Unterstützung des Prozesses im Vordergrund steht (P1).

Dazu realisiert die in Abb. 3 dargestellte grundlegende Aufteilung eine einheitliche und wiederkehrende Oberflächengestaltung über verschiedene Darstellungstypen in der Prozessführung hinweg. Hier dient die Statusleiste im oberen Bereich des Bildschirms der Anzeige ausgewählter System- und Anwendungsinformationen. Der Status der Spracherkennung und die Uhrzeit werden durchgehend ange- 
zeigt, wohingegen Informationen zur WLAN-Verbindung und zum Akkustand nur bei Handlungsbedarf auftauchen.

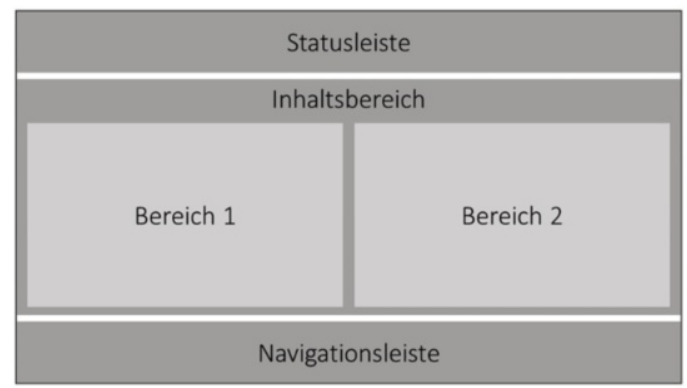

Abb. 3. Aufteilung der Oberfläche

Im Hauptbereich der Anwendung werden die Kerninformationen und Anweisungen zur Bearbeitung des jeweiligen Arbeitsschritts platziert. Der Inhaltsbereich wird für die Umsetzung von Darstellungstypen mit verschiedenen Informationstypen in zwei Teilbereiche getrennt. So wird bspw. die parallele Anzeige von bildlichen und textuellen Informationen für verschiedene Anwendungsszenarien standardisiert. Dabei wird, entsprechend der in Abschnitt 3 beschriebenen Erkenntnisse, das Bild als zentrale Informationsquelle auf der inneren Bildschirmhälfte platziert.

Zuletzt befindet sich am unteren Bildschirmrand eine Navigationsleiste, die eine Anzeige zum Prozessfortschritt und die Schaltflächen zur Vorwärts- und Rückwärts-Navigation beinhaltet. Dadurch hat der Anwender jederzeit einen Überblick zu seiner aktuellen Position in der Prozessführung, womit die Umsetzung von (P3) unterstützt werden soll.

Um die vorhandenen Erkenntnisse zur Farbgebung von Smart-Glasses-Anwendungen umzusetzen, wird die Oberfläche von einem schwarzen Hintergrund geprägt, worauf Bilder und helle Schrift angezeigt werden. Dabei wird zwischen textuellen Informationen in weißer Farbe und verfügbaren Sprachbefehlen mit Anführungszeichen und in hellblauer Farbe differenziert. Diese Farbgebung der Sprachbefehle wurde auf hellblau als neutrale, aber gut sichtbare Farbe festgelegt, da Signalfarben ausgeschlossen wurden. Dagegen wurden in der Statusleiste bewusst Signalfarben verwendet, um auf gegebenen Handlungsbedarf hinzuweisen. Der Status der Sprachsteuerung wird mit einer farblichen Kodierung, angelehnt an die Ampelfarben, übermittelt: Ein grünes Symbol vermittelt die Bereitschaft für einen Sprachbefehl, ein gelbes Symbol bedeutet die aktuelle Verarbeitung eines Sprachbefehls und ein rotes Symbol weist auf einen Fehler hin.

Dieses UI-Konzept entstand im Rahmen der iterativen, prototypischen Umsetzung, sodass die Weiterentwicklung an Rückmeldungen und Bedürfnissen der Anwender ausgerichtet war. 


\section{Implementierung des Informationssystems}

Für die prototypische Implementierung wurde eine klassische Client-Server-Architektur ausgewählt (vgl. Abb. 4). Neben der Anbindung an das eigenständige Datenbanksystem nach dem REST-Konzept wäre eine Integration in ein bestehendes ERP-System möglich. Der Prototyp wurde in der Entwicklungsumgebung Android Studio von Google für die Android Version 6.01 mit dem API Level 23 umgesetzt.

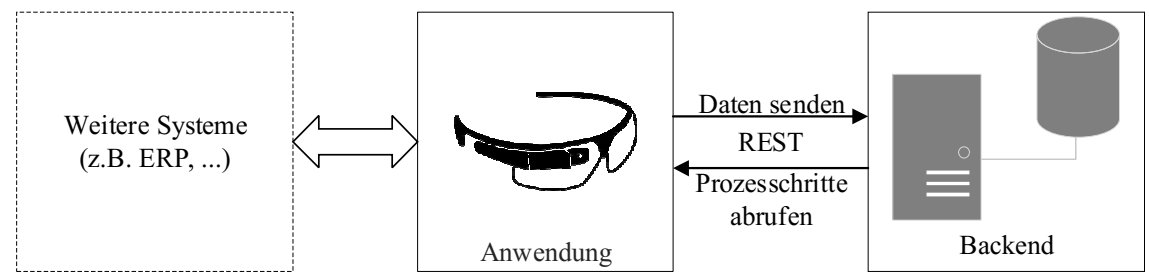

Abb. 4. Client-Server-Architektur

\subsection{Implementierung der Anwendung zur Prozessführung}

\subsubsection{Aufbau der Anwendung}

Bei dem Entwurf und der Umsetzung der Anwendung wurde ein möglichst hoher Grad der Modularisierung und Generalisierung angestrebt. Die Klassenhierarchie ist in Abb. 5 dargestellt.

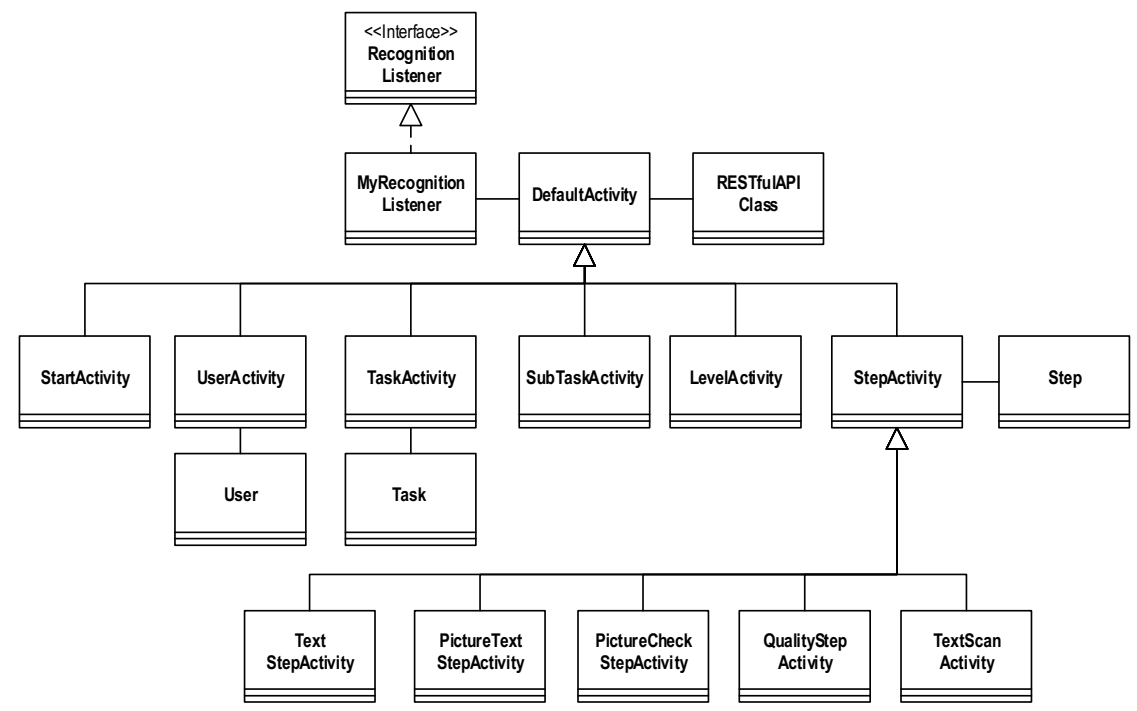

Abb. 5. Klassenhierarchie der Anwendung 
Die DefaultActivity fungiert als Oberklasse und beinhaltet die Konfiguration und Steuerung der Interaktionsmöglichkeiten sowie die Kommunikation zum Backend. Die Klassen StartActivity, UserActivity, TaskActivity, SubTaskActivity sowie LevelActivity implementieren die Prozessinitialisierung. Bspw. wurde die Benutzeranmeldung in der Anwendung durch den Scan eines Barcodes umgesetzt. In diesem Anwendungsabschnitt wären weitere anwenderabhängige Einstellungen und Individualisierungen denkbar, z. B. ein an Farbenblinde angepasstes Farbschema.

Die StepActivitiy erweitert die DefaultActivity um die Prozessführungsfunktionalität. Dazu wurde die Logik der dynamischen Schrittabfolge und damit die Steuerung aufeinanderfolgender Prozessschritte und ihrer Darstellungstypen umgesetzt. Außerdem wird die Navigationsleiste für alle StepActivities gesteuert. Entsprechend der in Abschnitt 4.2 vorgestellten Darstellungstypen wurden vier Spezialisierungen der StepActivity umgesetzt.

Um die Kommunikation mit dem Backend umzusetzen, wurde die Klasse RESTfulAPIClass erstellt. Diese verwaltet den Datenaustausch und bereitet die empfangenen Daten entsprechend auf. Mit der Verwendung des REST-Konzeptes ist eine standardisierte und sichere Kommunikation gewährleistet. Die Klassen User, Task und Step dienen dazu, die Daten in der Anwendung zu organisieren.

\subsubsection{Ablauf der Anwendung}

Ein beispielhafter Ablauf der Anwendung ist in Abb. 6 dargestellt. Die Prozessinitialisierung und -führung wurden auf Grundlage der in Abschnitt 4.2 formulierten Anforderungen umgesetzt. In der Prozessinitialisierung werden die Anmelde- und Aufgabenauswahlschritte als reine Textanweisungen dargestellt. Die konkrete Anmeldung und Auswahl erfolgen via Barcode-Scan. Der Anwender bekommt anschließend eine kurze Übersicht über den aktuellen Auftrag. Die Auswahl der Teilaufgabe und der Kompetenzstufe erfolgt über Listen (A3 und A4). So werden je nach ausgewählter Aufgabe zugehörige Teilaufgaben aufgelistet und verschiedene Kompetenzstufen angeboten. Die Bezeichnungen der Teilaufgaben und Kompetenzstufen werden automatisch in Sprachbefehle transformiert.

Für die Prozessführung wurden die abgeleiteten Darstellungstypen umgesetzt. Dabei stand die Umsetzung des dynamischen Ablaufs sowie die Implementierung einer intuitiven Qualitätskontrolle (A5) im Vordergrund. Da die Prozessführung abhängig von Nutzereingaben angepasst werden muss, wird eine Kontrollmaske verwendet. Über die Auswahl einer Ja/Nein-Antwort können darin Abweichungen vom normalen Prozessfluss angegeben werden. Damit kann bspw. auf das Fehlen eines benötigten Artikels reagiert oder der erfolgreiche Abschluss eines Arbeitsabschnitts abgefragt werden. Neben der Umsetzung der Qualitätskontrolle wurden einfache Arbeitsanweisungen implementiert. Diese geben dem Anwender Textund Bildanweisungen und haben bis auf die Navigationselemente keine Interaktionsmöglichkeiten. Nach Abschluss des Auftrages kann der Anwender einen weiteren Auftrag auswählen. 


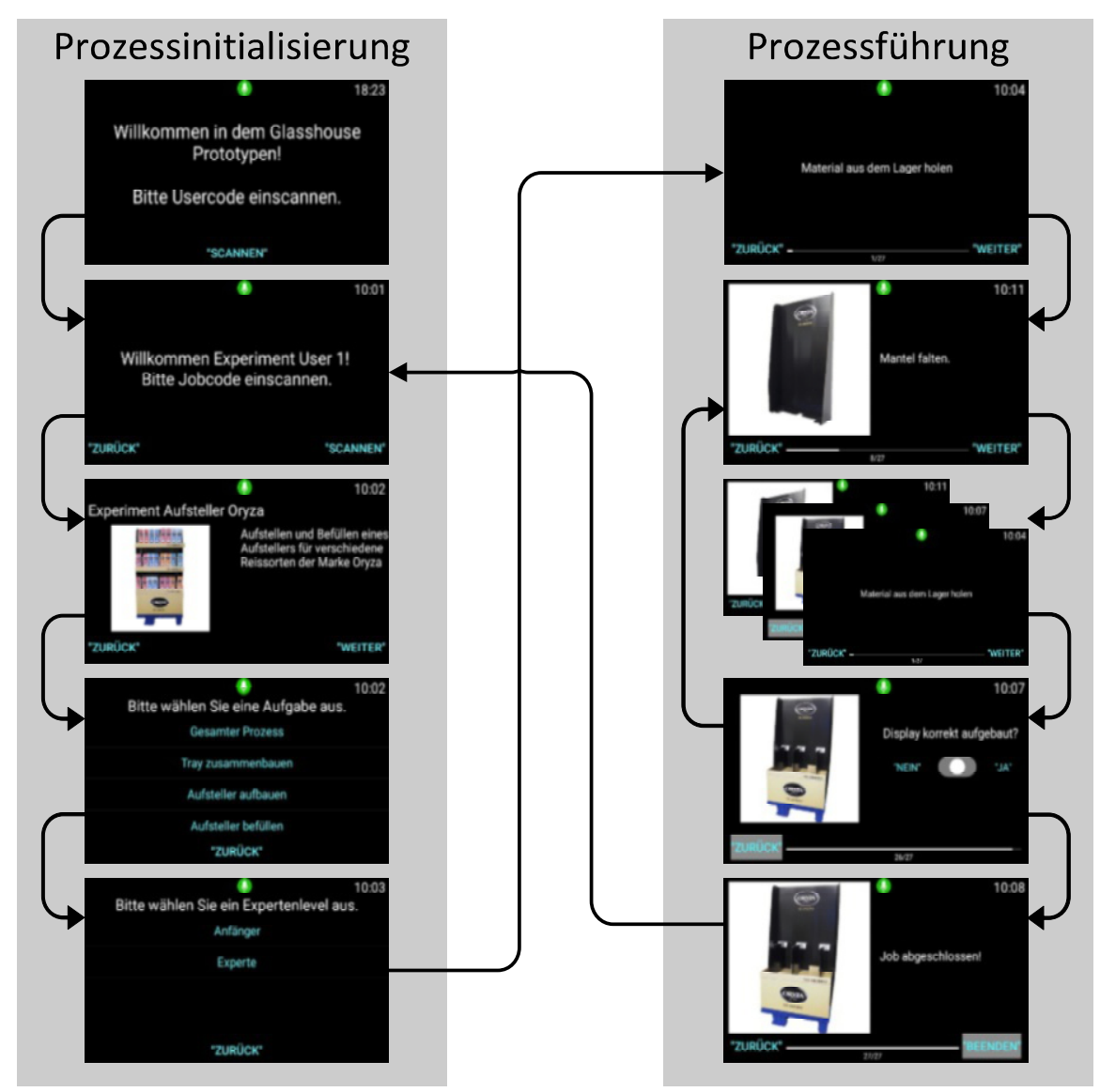

Abb. 6. Umsetzung des Anwendungsablaufs

\subsubsection{Implementierung des Graphical User Interface}

Das im Prototyp verwendete Layout richtet sich nach dem konzipierten Design, welches in Abschnitt 4.3 beschrieben wurde. In der Umsetzung wurden von der generischen Oberflächenaufteilung mehrere Layouts für die unterschiedlichen Darstellungstypen abgeleitet. Die einzelnen Layouts unterscheiden sich in ihrer Nutzung des zentralen Inhaltsbereichs, welcher abhängig vom Darstellungstyp die Informationen anzeigt. 


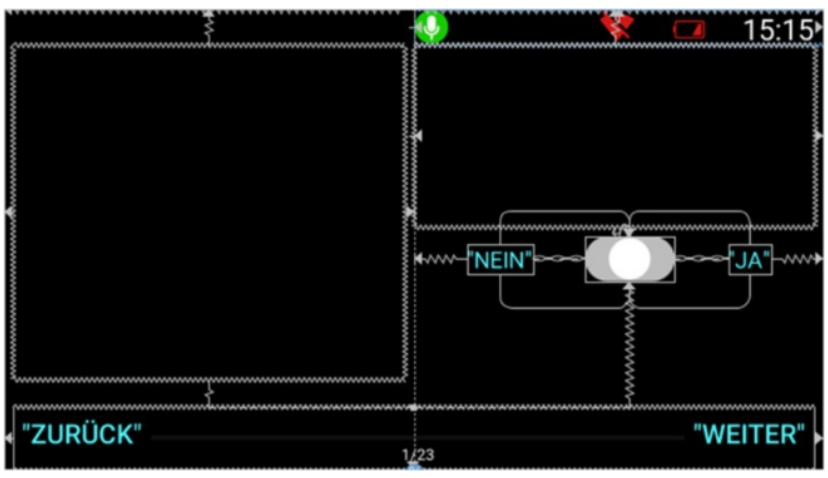

Abb. 7. Layout für einen Schritt mit Kontrollfunktion

In Abb. 7 ist beispielhaft das Layout für einen Schritt mit Kontrollfunktion dargestellt. Darin wird die Verankerung der Elemente in einem ConstraintLayout festgelegt, welche in der Abbildung durch Linien visualisiert ist. So wird ein responsives Design erreicht, womit die Anwendung auf andere Endgeräte übertragbar ist.

Die von Android bereitgestellte Statusleiste enthält zu vernachlässigende Informationen, die bei der Verwendung mit der Smart Glasses zudem nicht ausreichend wahrnehmbar sind. Daher wurde eine an den Anwendungsfall angepasste Statusleiste mit einer für Smart Glasses angemessenen Größe implementiert. Diese wurde im oberen Bereich angeordnet und enthält Symbole für den Status der Spracherkennung, die WLAN-Verbindung und den Akkustand sowie die Uhrzeit. Sämtliche Layouts beinhalten im unteren Bereich eine Navigationsleiste, welche die Vor- und Rückwärts-Navigation und bei der Prozessführung eine Fortschrittsleiste (A2) beinhaltet. Die Navigation wurde in Form von Buttons implementiert, sodass sowohl die Sprachsteuerung als primäre Interaktionsform, als auch eine Betätigung durch die Hardwaretasten ermöglicht werden. Der Fortschritt wurde durch eine lineare Leiste umgesetzt, welche die dynamische Prozessführung nicht immer angemessen abbilden kann. Durch die Nutzereingaben in der Kontrollfunktion können bspw. Schritte übersprungen oder wiederholt werden.

Der zentrale Inhaltsbereich ist im Layout für Schritte mit Kontrollfunktion in zwei Bereiche unterteilt. Im linken Bereich werden Kerninformationen in bildlicher Form dargestellt. Die rechte Seite beinhaltet im oberen Teil einen weiteren kleinen Informationsbereich, der primär für die Darstellung kurzer, textueller Anweisungen konzipiert wurde. Hier werden zumeist Fragen angezeigt, die als Kontrolle vom Nutzer zu beantworten sind. Darunter befindet sich ein Schieberegler, der anfänglich mittig steht und einen neutralen Zustand hat, sodass der Befehl „Weiter“ beim Aufruf des Schrittes noch nicht ausgeführt werden kann. Erst wenn der Nutzer die Kontrolle abgeschlossen und somit den Schieberegler auf „Ja“ oder „Nein“ bewegt hat, wird der nächste Befehl (,Weiter” bzw. „Zurück“) sichtbar und nutzbar. 


\subsubsection{Implementierung der Qualitätskontrolle}

Es wurden zwei verschiedene Arten der Qualitätskontrolle implementiert (A5). Eine einfache Kontrolle nutzt das im vorangegangenen Abschnitt beschriebene Layout, bei der der Anwender den aktuellen Arbeitserfolg durch einen Bildabgleich bestätigen muss. Diese Kontrolle eignet sich für den Abschluss von Teilabschnitten im Prozess und der Sicherstellung eines Zwischenergebnisses.
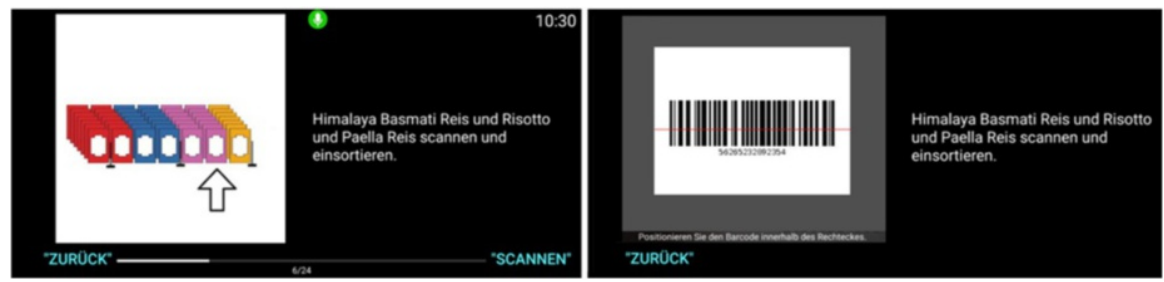

Abb. 8. Zweischrittige Qualitätskontrolle

Zusätzlich gibt es eine komplexere Kontrollfunktion mit einem zweischrittigen Ablauf, der in Abb. 8 dargestellt ist. Hier wird zunächst eine Anweisung mit Bildabgleich angezeigt, deren Ausführung anschließend mit einem Barcode-Scan bestätigt werden muss. Dies ist speziell auf den Anwendungsfall zugeschnitten, bei dem Artikel verpackt und einsortiert werden. Neben der Sicherstellung, dass der korrekte Artikel genommen wurde, kann der Artikel direkt aus dem ERP-System ausgebucht werden und weitere Prüfungen, wie zum Beispiel auf ein ausreichendes Mindesthaltbarkeitsdatum, können automatisch vorgenommen werden.

\subsection{Implementierung der Interaktionsmöglichkeiten}

Die Möglichkeit zur freihändigen und intuitiven Interaktion mit dem Informationssystem gehört zu den Vorteilen der Unterstützung des Kommissionierungsprozesses durch eine Smart-Glasses-Plattform gegenüber der klassischen Papieranleitung. Dazu wurden verschiedene Interaktionsarten implementiert und ihre Funktionalität wird im Folgenden erläutert.

\subsubsection{Sprachsteuerung}

Primäres Mittel zur Umsetzung einer freihändigen Interaktion bildet die Sprachsteuerung. Für die Smart Glasses Vuzix M300 gibt es eine vom Hersteller integrierte Sprachsteuerung, die auf die Erkennung einzelner Befehle ausgelegt ist. Dabei können die Standardbefehle mithilfe eines SDK um selbst gewählte Befehle in verschiedenen Sprachen erweitert werden. Im Rahmen der iterativen Evaluation des Prototyps hat sich die Erkennung und Verarbeitung zumindest deutscher Befehle als nicht zufriedenstellend erwiesen. 
Daher wurde eine alternative Umsetzung angestrebt, an die folgende Anforderungen gestellt wurden: Offline-Verfügbarkeit, Schnelligkeit, Kompatibilität, Verwendbarkeit selbsterstellter Befehle und Kostenfreiheit.

Nach einer Bewertung der zugänglichen Lösungen wurde die Google Speech Recognition gewählt. Diese Lösung ist jedoch mit Limitationen verbunden, da der Google Service eher auf eine Spracherkennung, als eine Sprachsteuerung ausgelegt ist, sodass die Funktionalität nicht auf das Herausfiltern einzelner Befehle optimiert ist. Dennoch ist mit dieser Herangehensweise eine weitgehend zuverlässige Erkennung und Verarbeitung von Sprachbefehlen möglich (A1).

Die Basis der Sprachsteuerungsfunktion sowie ihre Statusanzeige wird in der Oberklasse DefaultActivity implementiert. Alle erbenden Klassen müssen somit lediglich in der Methode handleResult die für ihre spezifische Funktionalität notwendigen Sprachbefehle (z.B. „scannen”) abfangen und passende Reaktionen definieren (z.B. einen Scanvorgang starten).

\subsubsection{Hardware-Tasten und Touchpad}

Das Modell M300 umfasst drei Hardware-Tasten und ein Touchpad als manuelle Steuerungsmöglichkeiten. Allerdings ist weder die Belegung dieser Tasten intuitiv, noch ist ihre Handhabung im praktischen Gebrauch günstig. Zum einen werden je nach Länge des Tastendrucks unterschiedliche Funktionen ausgeführt, zum anderen kann durch Verwenden der Tasten die Smart Glasses verrutschen. Dies bestätigt den Bedarf der freihändigen Interaktion, die mit diesem Prototyp angestrebt wird (A1). Dennoch wird die Navigation innerhalb der Anwendung auch mithilfe der Hardware-Steuerungselemente ermöglicht, da bei bestimmten Störeinflüssen, bspw. lauten Umgebungsgeräuschen, die Funktionsfähigkeit der Sprachsteuerung eingeschränkt sein kann.

\subsubsection{Kamera}

Neben den Hardwaretasten und dem Touchpad verfügt die M300 auch über eine eigene Kamera. Diese lässt sich für unterschiedliche Aufgaben, wie bspw. das Erkennen von Barcodes oder das Aufnehmen von Bildern nutzen.

Die Kamera wurde gezielt genutzt, um Barcodes aufzunehmen und sich anhand dieser im Prozess fortzubewegen. Dabei wurde eine geringe Dauer der Kameranutzung angestrebt, um den Akkuverbrauch zu reduzieren und die Privatsphäre der Nutzer zu schützen. Die Kamera wird in Voll- und Teilbild eingesetzt. Das Teilbild wird für Vorgänge verwendet, bei denen zusätzliche Informationen, Bildabgleiche oder Handlungsanweisungen parallel angezeigt werden. Auch die Anzeige eines Videos, das die korrekte Durchführung des Schrittes darstellt, wäre denkbar. Für simple Vorgänge wird der Vollbildmodus verwendet.

\subsubsection{Externe Peripherie}

Im Rahmen der prototypischen Entwicklung wurde das Myo-Armband als externe Peripherie eingebunden, welches per Bluetooth mit der Smart Glasses verbunden 
wird. Das Armband erkennt, welche Muskeln für bestimmte Gesten beansprucht werden und muss dafür nach jedem Anlegen am Oberarm des Nutzers kalibriert werden. Die Standardgesten umfassen ein Winken nach links und rechts, das Ballen der Faust, das Spreizen der Finger und das Tippen des Daumens gegen den Mittelfinger.

Zur Implementierung liefert Myo eine eigene Bibliothek mit, welche das Erkennen und Interpretieren der Standardgesten ermöglicht. Für den Prototyp wurden die Gesten des Winkens nach rechts und links ausgewählt, um intuitiv durch den Prozess zu navigieren. Dabei wird ein Winken nach links so interpretiert, dass man mit dem nächsten Schritt fortfahren möchte, ein Winken nach rechts ruft den vorangegangenen Schritt erneut auf.

Das Myo-Armband erweitert die Interaktionsmöglichkeiten, weist jedoch auch einige Limitationen auf. So ist die Kalibrierung bei jedem Anlegen umständlich und das Tragen das Armbands kann als unangenehm empfunden werden. Zudem ist die Gestenerkennung fehleranfällig, auch wenn die Ausführung nur leicht von den kalibrierten Gesten abweicht. Des Weiteren geht durch die Verwendung des Myo-Armbands der Nutzen der freihändigen Bedienung verloren (A1).

\section{Fazit und Ausblick}

Das Tool zur Kommissionierung auf einer Smart-Glasses-Plattform ermöglicht eine dynamische Prozessführung mit integrierter Qualitätssicherung. Dabei wurden eine freihändige Bedienung und intuitive Interaktion umgesetzt. Im Vergleich zur Papieranleitung konnten damit eine Verringerung des Zeitaufwands sowie eine Anpassung an die Kompetenz der Mitarbeiter realisiert werden. Zudem ist die Integration in ein bestehendes ERP-System möglich.

Die Umsetzung dieses Prototyps erfolgte unter Berücksichtigung der noch vorhandenen technischen Limitationen von Smart Glasses, bspw. einer beschränkten Akkulaufzeit oder Displaygröße. Durch die Entwicklung der Smart-Glasses-Technologie könnten diese Limitationen zukünftig aufgehoben werden. Die Erkenntnisse dieses Prototyps können daher nicht in ihrer konkreten Form weiterverwendet werden, sondern dienen als beispielhafte Konzeption und Umsetzung für folgende Projekte. Somit konnten, basierend auf den bei der Entwicklung dieses Prototyps gewonnenen Erkenntnissen, sowie schon etablierten Designgrundlagen, neue Guidelines für die Entwicklung für Smart Glasses erarbeitet werden (Berkemeier et al. 2019). Zukünftige Arbeiten müssen diese Guidelines evaluieren und könnten diese Ansätze für die Umsetzung von Smart-Glasses-basierten Informationssystemen auf weitere Anwendungsgebiete übertragen. 


\section{Literatur}

Berkemeier L, Zobel B, Werning S, Ickerott I, Thomas O (2019) Engineering of Augmented Reality-Based Information Systems. Business \& Information Systems Engineering 61:67-89

Google Inc. (2015) Design for Glass. https://developers.google.com/glass/design/, abgerufen am 08.01.2019

Horstmann F, Lingenfelder M (2015) POS-Marketing-Verbesserung durch die Integration von Dienstleistern in die Hersteller-Handels-Zusammenarbeit am Beispiel von Display-Promotions. In: Bruhn M, Hadwich K (Hrsg) Interaktive Wertschöpfung durch Dienstleistungen: Strategische Ausrichtung von Kundeninteraktionen, Geschäftsmodellen und sozialen Netzwerken. Forum Dienstleistungsmanagement. Springer Fachmedien, Wiesbaden, 541-566

Meta Company (2017) Interface Design. http://devcenter.metavision.com/design/userinterface-guidelines-interface-design, abgerufen am 08.01.2019

Niemöller C, Metzger D, Thomas O (2017) Design and Evaluation of a Smart Glassesbased Service Support System. In: Leimeister JM, Brenner W (Hrsg) Proceedings der 13. Internationalen Tagung Wirtschaftsinformatik (WI 2017). AISeL, St. Gallen, Schweiz, 106-120

Sony Corporation (2017) Design guidelines. https://developer.sony.com/develop/smarteyeglass-sed-e1/developer-tools/designguidelines, abgerufen am 08.01.2019

Tanuma K, Sato T, Nomura M, Nakanishi M (2011) Comfortable Design of Task-Related Information Displayed Using Optical See-Through Head-Mounted Display. In: Salvendy G, Smith MJ (Hrsg) Human Interface and the Management of Information. 418429

Vuzix Corporation (2017) Vuzix M300 Smart Glasses. https://www.vuzix.com/Products/CES-Products, abgerufen am 15.03.2019

Zobel B, Werning S, Berkemeier L, Thomas O (2018) Augmented- und Virtual-RealityTechnologien zur Digitalisierung der Aus- und Weiterbildung - Überblick, Klassifikation und Vergleich. In: Thomas O, Metzger D, Niegemann H (Hrsg) Digitalisierung in der Aus- und Weiterbildung. Springer Gabler, Berlin, Heidelberg 